\title{
PRODUÇÃO CIENTÍFICA BRASILEIRA EM GESTÃO DE OPERAÇÕES NO PERIODO 2000-2010
}

BRAZILIAN SCIENTIFIC PRODUCTION IN OPERATIONS MANAGEMENT IN THE PERIOD 2000-2012

PRODUCCIÓN CIENTÍFICA BRASILEÑA SOBRE GESTIÓN DE OPERACIONES EN EL PERÍODO 2000-2010

\section{RESUMO}

Este estudo analisa temas e métodos de pesquisa tratados por pesquisadores brasileiros da área de Gestão de Operações por meio de dois vetores complementares: a relevância e o rigor. Inicialmente, contemplaram-se os últimos 10 anos das principais revistas brasileiras da área de Administração. Complementarmente, analisaram-se periódicos internacionais mais relevantes da área de Gestão de Operações. Quanto aos temas mais frequen- tes nos artigos analisados, houve convergência entre o que foi publicado pelos autores brasileiros no Brasil e nos periódicos estrangeiros, com destaque para supply chain management e estratégia de operações. No que tange ao rigor metodológico, um dos principais desafios presentes é atentar para as mudanças em curso acerca de orientação metodológica dos estudos em Gestão de Operações no cenário internacional.

PALAVRAS-CHAVE Gestão de Operações, Brasil, métodos de pesquisa, produção científica, publicação.

Ely Laureano Paiva ely.paiva@fgv.br

Professor da Escola de Administração de Empresas de São Paulo, Fundação Getulio Vargas - São Paulo - SP, Brasil

Luiz Artur Ledur Brito luiz.brito@fgv.br

Professor da Escola de Administração de Empresas de São Paulo, Fundação Getulio Vargas - São Paulo - SP, Brasil

\begin{abstract}
The aim of this study is to analyze issues in Operations Management and the methodology employed by Brazilian researchers in this field. The analysis of the literature traced two complementary axes: relevance and rigor. We covered the last ten years by examining the main Brazilian journals in Business Administration and analyzing articles published in the main international journals in OM as well. The study found a certain level of convergence between the issues in the articles published by Brazilian authors in both groups of journals and the importance of supply chain management and operations strategies. The main challenge facing Brazilian researchers concerns the methodological changes in OM studies that are currently in progress on an international level.

keywords Operations Management, Brazil, research methods, scientific production, publication.

Resumen Este estudio analiza temas y métodos de investigación tratados por investigadores brasileños del área de Gestión de Operaciones, por medio de dos vectores complementarios: la relevancia y el rigor. Inicialmente, se contemplaron los últimos 10 años de las principales revistas brasileñas del área de Administración. Complementariamente, se analizaron los periódicos internacionales más relevantes del área de Gestión de Operaciones. Respecto a los temas más frecuentes en los artículos analizados, hubo convergencia entre lo que fue publicado por los autores brasileños en Brasil y en los periódicos extranjeros, principalmente supply chain management y estrategia de operaciones. En lo que se refiere al rigor metodológico, uno de los principales desafíos presentes es la atención a los cambios en curso acerca de orientación metodológica de los estudios sobre Gestión de Operaciones en el escenario internacional.

Palabras clave Gestión de Operaciones, Brasil, métodos de investigación, producción científica, publicación.
\end{abstract}




\section{INTRODUÇÃO}

A análise da produção científica pode ser abordada por meio de dois vetores complementares: a relevância e o rigor (GULATI, 2007). O primeiro ressalta a geração de conhecimento capaz de responder aos dilemas e desafios das organizações e da sociedade. Nesse aspecto, a publicação internacional da área de Gestão de Operações tem acompanhado o ambiente econômico global. Fatos como a emergência da produção enxuta, a integração crescente das cadeias de suprimentos e as pressões sociais por operações sustentáveis têm influenciado a temática da pesquisa nos países industrializados. Quanto ao rigor científico, vários fatores têm influenciado os métodos utilizados e a adequação destes às questões a serem respondidas.

Arkader (2003) e Correa, Paiva e Primo (2010) analisaram a produção científica no Brasil em Gestão de Operações. Há uma convergência de achados no que se refere aos temas preferenciais de pesquisa e às lacunas metodológicas. Prasad e Babbar (2000), ao estudar a temática de Gestão de Operações Internacionais, identificaram que os temas se modificaram ao longo dos anos, refletindo, de alguma maneira, o contexto socioeconômico à época da sua publicação. Desse modo, é possível que a área em Gestão de Operações no Brasil, ao longo das últimas décadas, também tenha direcionado suas discussões para temas considerados mais relevantes no contexto socioeconômico em que a publicação ocorreu. Explorando essas questões, este estudo propõe-se a analisar como os temas e os métodos de pesquisa têm sido tratados por pesquisadores brasileiros da área de Gestão de Operações.

\section{EPISTEMOLOGIA EM GESTÃO DE OPERAÇÕES}

Embora a área de Administração abrigue diferentes abordagens filosóficas (MILLER e TSANG, 2011), a área de Gestão de Operações teve sua evolução mais influenciada por um paradigma positivista, com origens em modelagem matemática e testes de hipóteses (SINGHAL e SINGHAL, 2012b). Filosofias da ciência como o realismo crítico, que reconhecem a existência de um mundo independente do conhecimento que o pesquisador tenha sobre ele, mas, ao mesmo tempo, reconhecem as limitações e falhas dos métodos de investigação, podem ajudar a área a evoluir. A ontologia do realismo crítico está baseada na existência de três domínios superpostos: o real, o vigente e o empírico (BHASKAR, 2008). O domínio real existe independentemente do observador e consiste nas relações potenciais entre os construtos. Essas relações são tendências que podem ou não se manifestar no domínio vigente. A cooperação com um fornecedor tem o poder de criar valor e benefícios para ambos (no domínio real), mas isso pode ou não acontecer no domínio vigente, dada a natureza complexa das relações causais num sistema aberto. O domínio empírico, por sua vez, consiste no que é observável com os métodos de investigação empregados. A relação entre cooperação e criação de valor pode existir de modo potencial no domínio real, manifestar-se no domínio vigente, mas não ser percebida completamente no domínio empírico, por não medirmos adequadamente os construtos, por exemplo.

Miller e Tsang (2011) propõem uma abordagem integrativa de investigação para teste de teorias, baseada no realismo crítico, composta de quatro etapas, ilustradas na Figura 1. A primeira etapa consiste na identificação, no plano teórico, das relações entre as variáveis $\left(\mathrm{x}_{1}\right.$ e $\mathrm{x}_{2}$ afetando y) e dos mecanismos $\left(\mathrm{m}_{1}\right.$ e $\mathrm{m}_{2}$ ) que explicam o processo pelo qual as relações ocorrem. Essa construção teórica está num sistema fechado, identificado pela elipse, e representa o domínio real. A segunda etapa consiste em verificar se os mecanismos hipotetizados realmente estão presentes no domínio vigente. O foco é testar a presença dos mecanismos. Estudos de caso com o uso de técnicas do tipo process tracing (GEORGE e BENNETT, 2005, p. 205-232) seriam especialmente adequados a essa tarefa. A terceira etapa consiste no teste das relações num ambiente fechado. Experimentos ou quasi-experimentos seriam técnicas adequadas. Finalmente, a última etapa é o teste do sistema teórico como um todo num ambiente aberto com um survey ou análises de dados secundários. O fato de o sistema ser aberto está representado pela ausência da elipse e pela introdução do termo $\varepsilon$, que agrupa o efeito de outras variáveis.

$\mathrm{Na}$ área de Gestão de Operações, como em outras, é frequente ver trabalhos de pesquisa que pulam as etapas 2 e 3 e vão diretamente da 1 para a 4 . Para fenômenos complexos como os que ocorrem nas ciências sociais, essa simplificação traz riscos relevantes à validade das conclusões.

O papel dos métodos qualitativos combinados com técnicas quantitativas tem sido debatido por outras áre- 
as e a área de Gestão de Operações pode saltar etapas ao se aproveitar deste debate. King, Keohane e Verba (1994) desenvolveram uma abordagem bastante fundamentada num paradigma quantitativo para a aplicação de métodos qualitativos. Esse trabalho abriu caminho para o desenvolvimento de abordagens mais balanceadas (BRADY e COLLIER, 2010), que são capazes de integrar de maneira harmônica as abordagens. Novos métodos, como o Qualitative Comparative Analysis (QCA), que usam dados qualitativos relacionados com uma lógica booleana (RIHOUX e RAGIN, 2009), eventualmente combinadas com conjuntos fuzzy (RAGIN, 2008), podem também ser uma oportunidade.

\section{PROCEDIMENTOS METODOLÓGICOS}

Para se responder ao objetivo deste estudo, foi realizado um levantamento que cobriu as publicações nacionais e internacionais de autores brasileiros na área, no período compreendido entre 2000 e 2010. Foram escolhidas revistas que possuem classificação A2 (Qualis/Capes), ligadas a associações ou a escolas da área de Administração e que publicam regularmente artigos de Gestão de Operações. Nesse primeiro grupo, foram analisadas a Revista de Administração Contemporânea (RAC), a RAE-Revista de Administração de Empresas (RAE) e a Brazilian Administration Review (BAR). Complementarmente, foram pesquisados periódicos internacionais mais relevantes da área de Gestão de Operações. Nesse grupo, foram incluídos os periódicos: Journal of Operations Mana- gement (JOM), Production and Operations Management (POM) e International Journal of Operations $\mathcal{E}$ Production Management (IJOPM). Acrescentou-se o International Journal of Production Economics (IJPE), que regularmente tem publicado estudos de autores brasileiros e fica na fronteira entre Administração e Engenharia de Produção.

\section{RESULTADOS}

\section{Produção em periódicos nacionais}

Ao longo da última década, foram encontrados 39 artigos sobre o tema Gestão de Operações nos três periódicos brasileiros. Há uma distribuição relativamente uniforme entre as duas publicações mais tradicionais ( $R A C$ e $R A E$ ). Vale lembrar que a revista $B A R$ iniciou suas atividades apenas em 2004 e, desse modo, a população de artigos pesquisados compreendeu apenas cinco anos.

Quanto aos temas, há a manutenção dos principais focos encontrados por Arkader (2003) e Correa, Paiva e Primo (2010), com destaque para supply chain management e estratégia de operações. Possivelmente, isso é decorrente da natureza desses temas, que permite interface com outras áreas de Administração. Desenvolvimento de Novos Produtos e Operações de Serviços aparecem como o terceiro e quarto temas mais estudados nas revistas nacionais. O crescimento do setor de serviços na economia mundial nas últimas décadas poderia justificar uma posição mais relevante para o tema. O mesmo poderia ser dito quanto a

\section{Figura 1 - Realismo crítico}

\begin{tabular}{|c|c|c|c|}
\hline Etapa 1 & Etapa 2 & Etapa 3 & Etapa 4 \\
\hline $\begin{array}{l}\text { - Identificar os mecanis- } \\
\text { mos hipotetizados. }\end{array}$ & $\begin{array}{l}\text { - Testar a presença dos } \\
\text { mecanismos m1 e m2 }\end{array}$ & $\begin{array}{l}\text { - Teste das relações cau- } \\
\text { sais de maneira isolada. }\end{array}$ & $\begin{array}{l}\text { - Teste do sistema teórico } \\
\text { em ambiente aberto. }\end{array}$ \\
\hline & $\begin{array}{l}\text { existência dos meca- } \\
\text { nismos, não sendo um } \\
\text { teste de relações. } \\
\text { - Estudos de caso seriam } \\
\text { adequados para tal. }\end{array}$ & & $X_{1}$ \\
\hline
\end{tabular}

Fonte: MILLER e TSANG, 2011 
desenvolvimento de produtos. Esse tema relaciona-se à necessidade de respostas mais rápidas, visando à adequação de processos e ao lançamento de produtos no mercado, característica que tem se acentuado com a busca de inovação como um diferencial competitivo. Há uma sinalização de tema que deverá ter interesse crescente, que é Operações Sustentáveis. Por fim, o tema Gestão da Qualidade tem perdido fôlego nas últimas décadas, apesar de ter sido amplamente explorado ao longo da década de 1990.

Ao se analisarem os métodos de pesquisa, há um equilíbrio entre os métodos survey e estudo de caso, com uma pequena vantagem para esse último. Vale ressaltar que o crescimento da utilização do método survey ocorreu notadamente na última década. Até então, havia um foco preferencial em estudos qualitativos baseados em estudos de caso. Essa situação, no Brasil, é oposta à dos Estados Unidos, onde a comunidade de pesquisa em Gestão de Operações, apenas na última década, tem se mostrado mais receptiva a estudos qualitativos.

\section{Tabela 1 - Publicações de autores brasileiros no Brasil (de 2000 a 2010)}

\begin{tabular}{|l|c|}
\hline \multicolumn{1}{|c|}{ Periódico } & $\begin{array}{c}\text { Número de } \\
\text { artigos com } \\
\text { autores } \\
\text { brasileiros }\end{array}$ \\
\hline Revista de Administração Contemporânea (RAC) & 18 \\
\hline Revista de Administração de Empresas (RAE) & 15 \\
\hline Brazilian Administration Review & 6 \\
\hline Total & 39 \\
\hline
\end{tabular}

\section{Tabela 2 - Temas preferenciais nos artigos publicados no Brasil (de 2000 a 2010)}

\begin{tabular}{|l|c|c|}
\hline \multicolumn{1}{|c|}{ Tema } & $\begin{array}{c}\text { Número de } \\
\text { artigos }\end{array}$ & Percentual \\
\hline Supply chain management & 20 & 51 \\
\hline Estratégia de operações & 5 & 13 \\
\hline $\begin{array}{l}\text { Desenvolvimento de novos } \\
\text { produtos }\end{array}$ & 5 & 13 \\
\hline Operações de serviços & 4 & 10 \\
\hline Gestão da qualidade & 3 & 8 \\
\hline Operações sustentáveis & 1 & 2,5 \\
\hline Ensino & 1 & 2,5 \\
\hline Total & 39 & 100 \\
\hline
\end{tabular}

Estudos de modelagem, técnica que, nos Estados Unidos, dominou amplamente o campo até a década de 1990, aparecem de modo limitado na publicação nacional. Possivelmente, essa orientação metodológica está mais presente em programas de Engenharia de Produção. Outros métodos, como estudos com dados secundários, também apareceram marginalmente.

\section{Produção internacional de autores brasileiros}

Todos os periódicos pesquisados possuem fatores de impacto representativos, e há uma histórica baixa participação de autores brasileiros nos três periódicos mais relevantes da área (JOM, POM e IJOPM). Não houve nenhuma publicação de autores brasileiros, no período pesquisado, no periódico $P O M$. No periódico com mais alto fator de impacto (JOM), foram encontrados dois artigos de autores brasileiros, hoje filiados a universidades nacionais, ambos em coautoria com autores estrangeiros. Se expurgarmos a edição especial sobre supply chain management na América Latina do periódico IJOPM, a participação de autores brasileiros é ainda tímida nesse periódico. No periódico IJPE, aparece uma participação mais expressiva de autores brasileiros. Várias explicações podem ser dadas para essa situação. Uma primeira seria o maior número de números publicados por ano pelo IJPE, sendo esta uma publicação mensal e as demais, bimestrais. A segunda explicação possível seria uma orientação editorial realmente internacional, que pode ser identificada pela heterogeneidade de países e de filiação a universidades dos autores. A terceira explicação, que pode ser decorrente das duas primeiras, é um nível de exigência menor, considerando-se que

\section{Tabela 3 - Métodos utilizados nos artigos publicados no Brasil (de 2000 a 2010)}

\begin{tabular}{|l|c|c|}
\hline \multicolumn{1}{|c|}{ Métodos } & $\begin{array}{c}\text { Número de } \\
\text { artigos }\end{array}$ & Percentual \\
\hline Estudos de caso & 19 & 49 \\
\hline Survey & 15 & 38,5 \\
\hline Modelagem & 2 & 5 \\
\hline Estudo quantitativo com & 1 & 2,5 \\
\hline dados secundários & 1 & 2,5 \\
\hline Artigo teórico & 1 & 2,5 \\
\hline Metaanálise & 36 & 100 \\
\hline Total & & \\
\hline
\end{tabular}


há mais edições por ano e há uma orientação internacionalizada da publicação, o que pode aumentar o percentual de artigos aceitos.

Os temas encontrados com maior frequência sugerem a presença de autores de escolas de Engenharia de Produção juntamente com autores de escolas de Administração. Essa é a explicação para a presença destacada de estudos que abordaram programação da produção e utilizaram modelagem matemática como método de pesquisa, situação não presente nos periódicos nacionais considerados. Por outro lado, os dois temas seguintes com ênfase mais destacada coincidem com o encontrado nas revistas brasileiras analisadas: supply chain management e estratégia de operações, confirmando os achados de Arkader (2003) e de Correa, Paiva e Primo (2010). Na sequência, apareceram temas ligados à inovação, como Desenvolvimento de Novos Produtos. O foco em Desempenho de Operações aparece, aqui, não necessariamente ligado à estratégia de operações. Foram enfocadas questões como giro de estoques, custo operacional e lead time, entre outros aspectos. Operações de Serviços aparece como um dos temas com crescente importância na área, refletindo o pa- pel do setor de serviços na economia atual. Por fim, é identificável a presença de Gestão de Qualidade, porém com decrescente importância, como ocorrido nos periódicos nacionais.

Quanto aos métodos, a presença de autores de programas de Engenharia de Produção influenciou o resultado encontrado. Há a liderança de estudos que utilizam Modelagem como método de pesquisa, seguidos dos estudos empíricos qualitativos e quantitativos. Tem-se, assim, uma situação próxima da encontrada nos Estados Unidos, em que essas duas grandes orientações (modelagem e estudos empíricos) dominam o campo de estudos em Gestão de Operações. No Brasil, os estudos empíricos são dominantes nos programas de Administração.

Quanto aos estudos empíricos, há um equilíbrio entre estudos quantitativos do tipo survey e qualitativos como estudos de caso, assim como identificado nos periódicos brasileiros. Ao mesmo tempo, aparecem outros métodos não encontrados nos periódicos do Brasil, como experimentos e pesquisa-ação. Estudos que utilizam pesquisa-ação remetem a uma tradição mais presente no Reino Unido, mas que, paulatinamente, tem conquistado espaço no Brasil e

\section{Tabela 4 - Publicações de autores brasileiros em periódicos internacionais (2000-2010)}

\begin{tabular}{|l|c|c|}
\hline \multicolumn{1}{|c|}{ Periódico } & $\begin{array}{c}\text { Fator de } \\
\text { Impacto }\end{array}$ & $\begin{array}{c}\text { Número de artigos com } \\
\text { Autores brasileiros }\end{array}$ \\
\hline journal of Operations Management & 5,093 & 2 \\
\hline International Journal of Operations and Production Management & 1,812 & 10 \\
\hline International Journal of Production Economics & 1,988 & 41 \\
\hline Total & & 53 \\
\hline
\end{tabular}

Tabela 5 - Temas preferenciais de autores brasileiros em periódicos internacionais (2000-2010)

\begin{tabular}{|l|c|c|}
\hline \multicolumn{1}{|c|}{ Tema } & Número de artigos & Percentual \\
\hline Programação da Produção & 13 & 23 \\
\hline Estratégia de operações & 9 & 17 \\
\hline Supply chain management & 9 & 17 \\
\hline Desenvolvimento de produtos & 8 & 15 \\
\hline Desempenho em operações & 5 & 9 \\
\hline Operações de serviços & 4 & 9 \\
\hline Gestão da qualidade & 3 & 6 \\
\hline Gestão da variedade de produtos & 2 & 4 \\
\hline Total & 53 & 100 \\
\hline
\end{tabular}


mesmo nos Estados Unidos. Em 2011, apareceu uma das primeiras publicações utilizando esse método de pesquisa em periódicos de destaque como o JOM (ver NAIR, MALHOTRA, AHIRE, 2011).

\section{Discussão sobre tendências em relação aos principais métodos}

Apesar do contínuo esforço dos pesquisadores brasileiros visando ao aumento de publicações internacionais, é necessário atentar para as tendências atuais nesses periódicos e ajustar tanto temas como abordagem metodológica. Reproduzir o padrão histórico de pesquisa pode não garantir a geração de pesquisas com alto potencial de publicação.

Há indicações de uma mudança de abordagem metodológica e epistemológica na área, principalmen-

\section{Tabela 6 - Métodos utilizados de autores brasileiros em periódicos internacionais (2000-2010)}

\begin{tabular}{|l|c|c|}
\hline Métodos & $\begin{array}{c}\text { Número de } \\
\text { artigos }\end{array}$ & Percentual \\
\hline Modelagem & 20 & 38 \\
\hline Survey & 15 & 28 \\
\hline Estudos de caso & 13 & 24 \\
\hline Experimento & 2 & 4 \\
\hline Artigo teórico & 2 & 4 \\
\hline Pesquisa-ação & 1 & 2 \\
\hline Total & 53 & 100 \\
\hline
\end{tabular}

te nos periódicos norte-americanos, para uma maior diversidade metodológica. Boyer e Swink (2008) já antecipavam essa mudança. Uma comparação, entre os anos de 2001 e 2011, dos tipos de artigos publicados no mais influente periódico da área, o JOM, evidencia essa mudança. Na Tabela 7 , observa-se um crescimento substancial de estudos de caso e de dados secundários, com um correspondente decréscimo da abordagem surveys. Esses dados refletem uma mudança ainda em curso, que tem sido discutida em palestras de pesquisadores líderes e painéis em eventos recentes. Singhal e Singhal resumem a necessidade dessa mudança: "a área de Gestão de Operações e da Cadeia de Suprimentos tem atualmente uma oferta excessiva de modelos e uma falta de teorias" (SINGHAL e SINGHAL, 2012a, p. 247, tradução nossa).

\section{Evolução da pesquisa tipo survey}

O uso de surveys atingiu um nível de sofisticação e rigorismo elevado, e o padrão dos artigos recentes do JOM (e.g. LIU e outros, 2012; MAHAPATRA, DAS, NARASIMHAN, 2012; ZACHARIA, NIX, LUSCH, 2011) pode ser tomado como referência para a área e campos relacionados, como marketing e estratégia. O trabalho de Mahapatra, Das e Narasimhan (2012), um exemplo típico, apresenta 322 respondentes, usa Q-sort, análise de viés de não respondentes, Common Method Variance (PODSAKOFF e outros, 2003), análise fatorial confirmatória antes do modelo estrutural, e finaliza com uma análise de multigrupos. Esse conjunto de técnicas e critérios é o padrão atual, distante da abor-

\section{Tabela 7 - Comparação dos artigos publicados no JOM nos anos 2001 e 2011}

\begin{tabular}{|l|c|c|c|c|}
\hline & \multicolumn{2}{|c|}{2001} & \multicolumn{2}{|c|}{2011} \\
\hline Survey & Artigos & Percentual & Artigos & Percentual \\
\hline Estudos de caso & 22 & 64 & 17 & 33 \\
\hline Dados secundários & 0 & 0 & 9 & 17 \\
\hline Experimentos & 3 & 9 & 14 & 27 \\
\hline Modelagem & 1 & 3 & 1 & 2 \\
\hline Múltiplos métodos & 2 & 6 & 2 & 4 \\
\hline Teóricos & 1 & 3 & 4 & 8 \\
\hline Outros/Metodológicos & 2 & 6 & 2 & 4 \\
\hline Total & 3 & 9 & 3 & 5 \\
\hline
\end{tabular}


dagem simplificada, ainda vista em alguns artigos no Brasil, baseados em amostras de conveniência de 100 respondentes utilizando regressão.

Embora as publicações brasileiras possam evoluir na direção desse padrão de qualidade, a sua conquista ainda não garante a publicação internacional futura. No jargão da área, esse padrão tornou-se um qualificador de pedidos. Praticamente todo pesquisador formado em programas de excelência em Gestão de Operações, nos Estados Unidos, tem uma capacitação que lhe dá proficiência nesse conjunto de técnicas. Os artigos publicados mais recentemente oferecem diferenciais metodológicos adicionais. São cada vez mais frequentes amostras de várias centenas de respondentes, chegando a 500, como em Mahapatra, Das e Narasimhan (2012). Pesquisas que enfrentam o problema do respondente único e, por exemplo, usam díades como unidade de análise (LIU e outros, 2012) podem ser outro fator diferenciador. Outras maneiras de oferecer um atrativo metodológico podem ser: a combinação com métodos qualitativos para validação dos questionários e interpretação dos resultados; o uso de métodos inovadores de coleta de dados (BLOOM e VAN REENEN, 2010); a análise multigrupos para avaliar efeitos de moderação (e.g. CAO e ZHANG, 2011); e o ainda inexplorado (na área) uso da teoria de resposta ao item na elaboração das escalas (MEADE e LAUTENSCHLAGE, 2004).

A pesquisa tipo survey continuará a ser uma técnica relevante para a área de Gestão de Operações, mas fica, para os brasileiros, o desafio de elevar o nível de suas abordagens metodológicas aos padrões internacionais. Assim, nossos programas de doutorado deveriam analisar se seus cursos de formação metodológica quantitativa capacitam os alunos para essas novas exigências.

\section{Estudos de caso e métodos qualitativos}

A tradição do uso de métodos de qualitativos, em especial, os estudos de caso, está mais estabelecida entre autores europeus. O IJOPM tem sido um periódico no qual alguns pesquisadores brasileiros têm publicado artigos com essa abordagem (FLEURY, 1999; PRIMO, DOOLEY, RUNGTUSANATHAM, 2007; WILK e FERSTENSEIFER, 2003). Recentemente, identifica-se um movimento, das principais publicações norte-americanas, de publicarem pesquisas com metodologias qualitativas incluindo estudos de caso, como evidenciado no caso da comparação 2001/2011 do JOM.
O elevado percentual de estudos de caso publicados nas revistas brasileiras em Gestão de Operações (19 dos 45 artigos) não garante, porém, que estejamos no caminho de termos mais chances de publicação internacional. A qualidade dos artigos nacionais baseados em estudos de caso tem bastante a evoluir, em relação aos artigos publicados recentemente pelo JOM. A análise de Barratt, Choi e Li (2011), expandindo artigos anteriores (MEREDITH, 1998; STUART e outros, 2002; VOSS, TSIKRIKTSIS, FROHLICH, 2002) sobre a evolução de estudos de caso na área de operações. pode ser um guia para os pesquisadores brasileiros se desenvolverem.

Barratt, Choi e Li (2011) dividem as abordagens dos estudos de caso em indutivas e dedutivas. Embora essa divisão seja, provavelmente, menos definida do que os autores sugerem, e as técnicas possam ser usadas para ambas as finalidades (WOODSIDE, 2010, p. 11), a divisão é útil para se entender o espectro de abordagens.

Os estudos de caso indutivos têm, por objetivo principal, a criação de teoria usando o processo de indução, e têm sido a abordagem predominante na área, tanto no plano internacional como no nacional. As referências clássicas (YIN, 2009; EISENHARDT, 1989; MEREDITH, 1998), relacionadas a essa abordagem, são as mais usadas nos trabalhos brasileiros. Um dos primeiros desafios é conciliar a justificativa da abordagem, a questão de pesquisa e o papel da teoria existente. É frequente ver-se, nos estudos brasileiros, a justificativa de que, como a pesquisa é exploratória, o estudo de caso seria recomendado. Essa justificativa é demasiadamente simples e, muitas vezes, incompleta. Há várias maneiras de desenvolver estudos exploratórios, inclusive com métodos quantitativos. Se o objetivo for criar ou desenvolver teoria, tem-se que demonstrar, de maneira extensiva, que não existe teoria adequada. A contribuição da teoria existente pode variar desde ser praticamente ausente, no caso da grounded theory, em que ela é extraída dos dados, até servir de pano de fundo, com o uso de construtos a priori para analisar os dados (EISENHARDT, 1989; MCCUTCHEON e MEREDITH, 1993). De qualquer forma, a identificação da lacuna teórica tem que estar clara - não pode ser uma lacuna empírica apenas - e a questão de pesquisa deveria estar associada a essa lacuna. Não basta termos uma pergunta de "como", se houver teorias existentes que podem explicar o "como". Fica a reflexão sobre se essa tarefa de construção de teoria é apropriada para dissertações de 
mestrado ou mesmo trabalhos de iniciação científica, como é frequente ocorrer no Brasil.

Além da questão da aplicabilidade, existe a questão do rigor metodológico no desenvolvimento e na apresentação dos resultados. Barratt, Choi e Li (2011, p. 330 e 331) oferecem um guia atualizado sobre a maior parte desses aspectos, cobrindo a definição da unidade de análise, seleção e número de casos, coleta e análise dos dados, organização dos resultados e análises dentro de cada caso e entre casos. A análise de publicações de estudos de caso nos números recentes do JOM (e.g. WU e PAGELL, 2011; WILHELM, 2011; LEWIS e BROWN, 2012) pode ser um guia prático.

Os estudos de caso chamados de dedutivos por Barrat, Choi e Li (2011) têm por objetivo o teste de teorias e são muito menos frequentes na área de Gestão de Operações. Apenas 35 artigos foram classificados como estudos de caso dedutivos, contra 169 indutivos na pesquisa feita por Barratt, Choi e Li (2011, p. 335). Os autores identificam duas alternativas básicas para usos de casos dedutivos: teste de teorias alternativas e estudos longitudinais. Entretanto, é possível pensar em outros usos dos estudos de casos dedutivos, combinando-os com técnicas quantitativas. Os testes de teorias nas ciências sociais apresentam complexidade elevada. Tem-se que enfrentar, por exemplo, características de equifinalidade (múltiplos caminhos causais para o mesmo resultado), multifinalidade (vários resultados possíveis com base no mesmo valor específico de uma variável), efeitos de retroalimentação, dependência de caminho, múltiplas interações, condições apenas necessárias para um resultado satisfatório (GEORGE e BENNET, 2005, p. 9). Como esperar que uma análise quantitativa que mede apenas relações lineares numa amostra que combina esses processos, com um número limitado de controles, nos ofereça um teste adequado? A percepção das limitações dos métodos quantitativos tem sido relatada em obras recentes, que fazem uma reflexão crítica de pesquisadores renomados de uma longa tradição em pesquisa quantitativa. William Starbuck (2006, p. 144), em seu livro The Production of Knowledge, oferece exemplos práticos.

\section{Pesquisas com dados secundários e experimentos}

Hendricks e Singhal (2005) ficaram reconhecidos pelo uso de dados secundários para analisar interrupções na cadeia de suprimentos e seus efeitos no desempenho financeiro. Recentemente, podem ser encon- trados vários artigos que exploram bancos de dados proprietários de empresas ou órgãos públicos cada vez mais ricos (e.g. GRIFFIS e outros, 2012; WAN, EVERS, DRESNER, 2012; QUEENAN, ANGST, DEVARAJ, 2011). Essa abordagem requer uma aproximação com o mundo empresarial, muito desejável por outros aspectos. Contrariamente ao senso comum, o Brasil dispõe de ricas bases de dados tanto em associações de classe como em órgãos do governo, apesar do pouco uso desta abordagem (DUARTE e outros, 2011)

O uso de experimentos tem uma tendência clara de crescimento, associado à crescente popularidade da abordagem comportamental da Gestão de Operações (GINO e PISANO, 2008). Embora ainda ausente nas publicações nacionais, essa abordagem tem aparecido em publicações internacionais (e.g. LETMATHE, SCHWEITZER, ZIELINSKI, 2012; VENKATESH, CHAN, THONG, 2012), confirmando sua anunciada emergência (BENDOLY, DONOHUE, SCHULTZ, 2006; BOYER e SWINK, 2008). Novamente, é uma clara oportunidade a ser explorada pelos pesquisadores brasileiros.

\section{CONCLUSÕES}

Se analisarmos os temas mais usuais na área de Gestão de Operações nos artigos analisados, há uma grande proximidade entre o que foi publicado no Brasil e nos periódicos estrangeiros, indicando um certo nível de convergência, excetuando-se o tema de programação da produção.

Pode-se considerar o ambiente empresarial como influenciador dos temas explorados pelos artigos da área de Gestão de Operações, agindo o pesquisador como um "intérprete bilíngue" capaz de dialogar com o mundo gerencial (GULATI, 2007). Nesse contexto, a estabilidade econômica do Brasil após o Plano Real tem levado ao aumento das atenções para setores que tem apresentado crescimento relevante. Entre estes, podem ser citados agronegócios, indústria química e automobilística, que apareceram com destaque entre os artigos analisados com foco em setores específicos. Também merece ser destacada a presença de estudos abordando serviços e a área de logística.

O setor de agronegócios, ainda que pesem as críticas quanto à tradição brasileira de exportador de commodities de baixo valor agregado, não pode ser menosprezado, principalmente pela presença global que algumas cadeias ganharam ao longo das últimas 
décadas, fazendo do Brasil um dos grandes produtores internacionais de produtos como carne bovina, carne de frango, soja, algodão, laranja, café e cana-de-açúcar. Entender como essas cadeias têm inovado, desenvolvido competências ou capabilities, utilizado tecnologias de informação, ou elevado sua integração com seus fornecedores (principalmente produtores rurais) são temas razoavelmente inexplorados. Também a relação desses aspectos com critérios de desempenho de operações, como custo, entrega e qualidade, são oportunidades presentes para a pesquisa na área de Gestão de Operações.

Várias lacunas ainda merecem ser respondidas também na indústria química, podendo-se citar a customização em produtos de fluxo contínuo e a inovação com foco em sustentabilidade como potenciais de vantagem competitiva. Ao mesmo tempo, os estudos do setor automobilístico, em vez de temas tradicionais, poderiam tentar entender qual estratégia de operações as empresas brasileiras estão desenvolvendo, já que há uma regionalização da produção global, que dividiria os mercados entre mercados mais sofisticados e emergentes. Enquanto Índia e alguns países do Leste Europeu miram uma estratégia de liderança em baixo custo no limite, qual posicionamento competitivo as empresas localizadas no Brasil estariam buscando?

Na cada vez mais relevante área de serviços, os temas preferenciais foram operações de varejo, setor bancário e setor de telecomunicações. Além destes, a área de gestão da saúde mereceria maior atenção, pois é um setor que tem apresentado destaque crescente na pesquisa internacional, pela sua importância socioeconômica. Percebendo a relevância desse setor, foi criado o College of Healthcare Management, filiado à associação Production and Operations Management Society. Nos artigos analisados, houve a ausência de textos com esse foco temático. Há, também, uma lacuna em estudos sobre o que tem sido chamado de operações humanitárias, internacionalmente motivados por fatos como o furacão Katrina, enchentes no Paquistão e tsunamis na Ásia. No Brasil, o recorrente problema de enchentes em diferentes regiões, como Sudeste e Norte, cria oportunidades para uma contribuição relevante da área de Gestão de Operações, com pesquisas que auxiliem a melhoria do tempo de resposta, diminuição dos custos envolvidos e logística de recursos em regiões atingidas.

A questão socioambiental continua relevante não apenas pelas pressões que os países têm feito, criando barreiras comerciais para produtos que suposta- mente não atendem às especificações, mas também pela escassez que se avizinha, em futuro próximo, de recursos até pouco considerados inesgotáveis, como a água. Entender melhor temas relacionados com cadeias de suprimentos fechadas (closed loop supply chains), desenvolvimento de produtos sustentáveis e condições de trabalho em setores de maior risco são, ainda, oportunidades de pesquisa com potencial de publicações internacionais pela crescente importância econômica do Brasil na arena global.

No que tange ao rigor científico, é necessário um esforço adicional para minimizar a lacuna histórica de inserção internacional. Desse modo, um dos principais desafios presentes é atentar para as mudanças de orientação metodológica em curso no cenário internacional. Inicialmente, as pesquisas baseadas tanto em survey como em métodos qualitativos precisam aproximar-se do nível de rigor metodológico atual das publicações de primeira linha internacional. Métodos menos utilizados, como experimentos, uso de dados secundários e pesquisa-ação, são também oportunidades. Esses métodos apresentam como vantagem potencial sua proximidade com o mundo empresarial. Ao mesmo tempo, também é importante o uso de múltiplas metodologias integradas, inseridas em projetos de pesquisa abrangentes. Fica o desafio para os nossos programas de mestrado e doutorado se inserirem nessa empreitada e formarem futuros pesquisadores capazes de desenvolver pesquisas com essas orientações.

\section{NOTA DE AGRADECIMENTO}

Agradecimentos aos bolsistas Márcia Regina Santiago Scarpin, Eliane Gomes da Costa e Fernando Gonçalves Picasso, pela colaboração.

\section{REFERÊNCIAS}

ARKADER, R. A pesquisa científica em gerência de operações no Brasil. RAE-Revista de Administração de Empresas, v. 43, n. 1, p. 70-80, 2003.

BARRATT, M; CHOI, T. Y; LI, M. Qualitative case studies in operations management: trends, research outcomes, and future research implications. Journal of Operations Management, v. 29, n. 4, p. 329-342, 2011. 
BENDOLY, E; DONOHUE, K; SCHULTZ, K. L. Behavior in operations management: assessing recent findings and revisiting old assumptions. Journal of Operations Management, v. 24, n. 6, p. 737-752, 2006.

BHASKAR. R. A realist theory of science. London; New York: Routledge, 2008.

BLOOM, N; VAN REENEN, J. New approaches to surveying organizations. American Economic Review, v. 100, n. 2, p. 105-109, 2010.

BOYER, K. K; SWINK, M. L. Empirical elephants: why multiple methods are essential to quality research in operations and supply chain management. Journal of Operations Management, v. 26, n. 3, p. 338-344, 2008.

BRADY, H. E; COLLIER, D. Rethinking social inquiry: diverse tools, shared standards. 2nd ed. Lanham: Rowman \& Littlefield, 2010. 410 p.

CAO, M; ZHANG, Q. Supply chain collaboration: impact on collaborative advantage and firm performance. Journal of Operations Management, v. 29, n. 3, p. 163-180, 2011.

CORREA, H. L; PAIVA, E. L; PRIMO, M. A. M. A pesquisa em gestão de operações no Brasil: um breve relato de sua evolução. RAE-eletrônica, v. 9, n. 2, p. 1-9, 2010. Disponivel em: http://www.scielo.br/pdf/raeel/v9n2/a02v9n2.pdf. Acesso em 22.10.2012.

DUARTE, A. L. C. M; BRITO, L. A. L; SERIO, L. C. D; MARTINS, G. S. Operational practices and financial performance: an empirical analysis of Brazilian manufacturing companies. BAR: Brazilian Administration Review, v. 8, n. 4, p. 395411, 2011.

EISENHARDT, K. M. Building theories from case study research. The Academy of Management Review, v. 14, n. 4, p. $532-550,1989$.

FLEURY, A. The changing pattern of operations management in developing countries: the case of Brazil. International Journal of Operations \& Production Management, v. 19, n. 6, p. 552-564, 1999.

GEORGE, A. L; BENNET, A. Case studies and theory development in social sciences. Cambridge: M.I.T, 2005. 331 p.

GINO, F; PISANO, G. Toward a theory of behavioral ope- rations. Manufacturing \& Service Operations Management, v. 10, n. 4, p. 676-691, 2008.

GRIFFIS, S. E; RAO, S; GOLDSBY, T. J; NIRANJAN, T. T. The customer consequences of returns in online retailing: an empirical analysis. Journal of Operations Management, v. 30, n. 4, p. 282-294, 2012.

GULATI, R. Tent poles, tribalism, and boundary spanning: the rigor-relevance debate in management research. Academy of Management Journal, v. 50, n. 4, p. 775782, 2007.

HENDRICKS, K. B; SINGHAL, V. R. An empirical analysis of the effect of supply chain disruptions on long-run stock price performance and equity risk of the firm. Production and Operations Management, v. 14, n. 1, p. 35-52, 2005.

KING, G; KEOHANE, R. O; VERBA, S. Designing social inquiry: scientific inference in qualitative research. Princeton: Princeton University, 1994. 245 p.

LETMATHE, P; SCHWEITZER, M; ZIELINSKI, M. How to learn new tasks: shop floor performance effects of knowledge transfer and performance feedback. Journal of Operations Management, v. 30, n. 3, p. 221-236, 2012.

LEWIS, M. A; BROWN, A. D. How different is professional service operations management? Journal of Operations Management, v. 30, n. 1-2, p. 1-11, 2012.

LIU, Y; HUANG, Y; LUO, Y; ZHAO, Y. How does justice matter in achieving buyer-supplier relationship performance? Journal of Operations Management, v. 30, n. 5, p. 355-367, 2012.

MAHAPATRA, S. K; DAS, A; NARASIMHAN, R. A contingent theory of supplier management initiatives: effects of competitive intensity and product life cycle. Journal of Operations Management, v. 30, n. 5, p. 406-422, 2012.

MCCUTCHEON, D. M; MEREDITH, J. R. Conducting case study research in operations management. Journal of Operations Management, v. 11, n. 3, p. 239-256, 1993.

MEADE, A. W; LAUTENSCHLAGE, G. J. A Comparison of item response theory and confirmatory factor analytic methodologies for establishing measurement equivalence/ invariance. Organizational Research Methods, v. 7, n, 4, p. 361-388, 2004. 
MEREDITH, J. Building operations management theory through case and field research. Journal of Operations Management, v. 16, n. 4, p. 441-454, 1998.

MILLER, K. D; TSANG, E. W. K. Testing management theories: critical realist philosophy and research methods. Strategic Management Journal, v. 32, n. 2, p. 139-158, 2011.

NAIR, A; MALHOTRA, M. K; AHIRE, S. L. Toward a theory of managing context in Six Sigma process-improvement projects: an action research investigation. Journal of Operations Management, v. 29, n. 5, p. 529-548, 2011.

PODSAKOFF, P. M; MACKENZIE, S. B; LEE, J.-Y; PODSAKOFF, N. P. Common method biases in behavioral research: a critical review of the literature and recommended remedies. Journal of Applied Psychology, v. 88, n. 5, p. 879903, 2003.

PRASAD, S; BABBAR, S. International operations management research. Journal of Operations Management, v. 18, n. 2, p. 209-247, 2000.

PRIMO, M. A. M; DOOLEY, K. J; RUNGTUSANATHAM, M. J. Manufacturing firm reaction to supplier failure and recovery. International Journal of Operations \& Production Management, v. 27, n. 3, p. 323-341, 2007.

QUEENAN, C. C; ANGST, C. M; DEVARAJ, S. Doctors' orders: if they're electronic, do they improve patient satisfaction? A complements/substitutes perspective. Journal of Operations Management, v. 29, n. 7-8, p. 639-649, 2011.

RAGIN, C. Redesigning social inquiry: fuzzy sets and beyond. Chicago: The University of Chicago, 2008. 225 p.

RIHOUX, B; RAGIN, C. C. Configurational comparative methods: qualitative comparative analysis (qca) and related techniques. Thousand Oaks: Sage, 2009.

SINGHAL, K; SINGHAL, J. Opportunities for developing the science of operations and supply-chain management. Journal of Operations Management, v. 30, n. 3, p. 245-252, 2012a.

SINGHAL, K; SINGHAL, J. Imperatives of the science of operations and supply-chain management. Journal of Operations Management, v. 30, n. 3, p. 237-244, 2012b.

STARBUCK, W. H. The production of knowledge: the challenge of social science research. New York: Oxford University, 2006. 208 p.

STUART, I; MCCUTCHEON, D; HANDFIELD, R; MCLACHLIN, $\mathrm{R}$; SAMSON, D. Effective case research in operations management: a process perspective. Journal of Operations Management, v. 20, n. 5, p. 419-433, 2002.

VENKATESH, V; CHAN, F. K. Y; THONG, J. Y. L. Designing e-government services: key service attributes and citizens' preference structures. Journal of Operations Management, v. 30, n. 1-2, p. 116-133, 2012.

VOSS, C; TSIKRIKTSIS, N; FROHLICH, M. Case research in operations management. International Journal of Operations E Production Management, v. 22, n. 2, p. 195219, 2002.

WAN, X; EVERS, P. T; DRESNER, M. E. Too much of a good thing: the impact of product variety on operations and sales performance. Journal of Operations Management, v. 30, n. 4, p. 316-324, 2012.

WILHELM, M. M. Managing coopetition through horizontal supply chain relations: linking dyadic and network levels of analysis. Journal of Operations Management, v. 29, n. 7-8, p. 663-676, 2011.

WILK, E. de O; FERSTENSEIFER, J. E. Use of resourcebased view in industrial cluster strategic analysis. Journal of Operations \& Production Management, v. 23, n. 9, p. 995-1009, 2003.

WOODSIDE, A. G. Case study research: methods, theory, practice. Bingley: Emerald, 2010. 440 p.

WU, Z; PAGELL, M. Balancing priorities: decision-making in sustainable supply chain management. Journal of Operations Management, v. 29, n. 6, p. 577-590, 2011.

YIN, R. K. Case study research: design and methods. 4th. ed. Thousand Oaks: Sage, 2009.

ZACHARIA, Z. G; NIX, N. W; LUSCH, R. F. Capabilities that enhance outcomes of an episodic supply chain collaboration. Journal of Operations Management, v. 29, n. 6, p. 591-603, 2011. 